\title{
Experimental studies of the efficient use of flexible tool in creep age forming
}

\author{
Qi Rong ${ }^{1, *}$, Yong $\mathrm{Li}^{1}$, Xiaoguang $\mathrm{Sun}^{2}$, Zhusheng Shi ${ }^{1}$, Lichun Meng ${ }^{2}$ and Jianguo Lin ${ }^{1}$ \\ ${ }^{1}$ Department of Mechanical Engineering, Imperial College London, London SW7 2AZ, UK \\ ${ }^{2}$ National Engineering Research Center for High-Speed EMU, CRRC Qingdao Sifang Co., Ltd, Qingdao 266111, China
}

\begin{abstract}
Application of a newly developed flexible forming tool to creep age forming (CAF) process has been investigated in this study. The flexible tool mainly consists of sparsely distributed forming pins, splines and elastomeric sheet. The effect of key factors related to the forming tool on the shape of the formed parts has been studied through various CAF experiments. The key factors investigated in this study include: the interval between forming pins, the arrangement of pins, the accuracy requirement of pin height and the material of splines. It has been found that reducing the interval between pins can efficiently smooth the shape of CAFed plates. The feasibility of asymmetric arrangement of pins has been proven, which can decrease the number of used pins, reduce tool weight, and increase efficiency. The forming results are very sensitive to the pin height, thus the experimental set-up error should be carefully controlled. Additionally, compared with mild steel, spring steel is more suitable as the spline material.
\end{abstract}

Keyword. Aluminium, Sheet metal, Flexible tool

\section{Introduction}

Creep age forming (CAF) is a forming method combing forming and age hardening in one process, and has become one of the most popular manufacturing methods for the production of extra-large panel components [1], such as upper wing skins of Airbus A380 [2], and Gulfstream G-IV Aircraft [3].

An important issue in the CAF process is the forming tool. One of the characteristic tools for various forming technologies is solid dies. However, the cost of solid dies for CAF is significant, as extra-large tools are generally required and tool modification is also frequently needed due to springback compensation. Levers [4-6] developed a semi-flexible forming tool for CAF of extra-large skin panels, replacing the solid dies into a set of rib boards and leading to the lighter weight and better flexibility than the solid dies, and its feasibility has already been proven to apply to the production of large aircraft panel components [7-9]. However, since the forming surface is dependent on the rib boards, when the shape of wing skin is changed, a new set of rib boards needs to be manufactured, which is costly and time-consuming. Thus, changing tool surface is a big challenge, which leads to the need of flexible tools for the CAF investigation, especially in a laboratory environment.

Lam et al. [10] have developed a multi-point flexible forming tool composed of sparsely distributed forming pins, splines, and elastomeric sheet. The forming pins

\footnotetext{
* Corresponding author: q.rong15@imperial.ac.uk
}

whose heights can be adjusted, are used to control the tool shape, splines are attached to the pins to maintain the continuity of the shape and an elastomeric sheet is used to more uniformly distribute the stress on the workpiece in the forming process. Compared to the tools mentioned above, this tool is much more flexible and with lower cost, making it a suitable choice for CAF study. However, it has been found that some kinks may occur on the deformed plates with this multi-point flexible tool when the target curvature of the workpiece is large and complex. Furthermore, how to use this tool in a more efficient way, i.e., using less pins and simpler arrangement to attain the required forming shape, is also of interest. The forming results will depend on each part of the tool, including forming pins, splines and elastomeric sheet, however no studies have been reported to investigate their detailed effects on the forming results currently.

For the forming pins, three factors are important to control the forming results: the interval between pins, increasing which can reduce the number of pins required and lower the tool weight; asymmetric arrangement of pins in top and bottom dies, which may largely decrease the number of pins required; and the accuracy requirement of pin height, which may affect the final forming shape. For the splines, the material used is also worth investigating, so as to obtain a smoother curved shape of the formed plate. 
Thus, in this study the effects of four different factors in the flexible forming tool on CAF forming results with aluminium alloy 6082 (AA6082) have been experimentally investigated, including the interval between neighbouring pins, asymmetric arrangement of pins, the error of pin height, and the material used for splines.

\section{Experimental programme}

\subsection{Experimental Material}

The material used in this study was AA6082-T6, whose chemical composition is listed in Table 1 . The as-received material had undergone solution heat treatment at $525^{\circ} \mathrm{C}$ for $22 \mathrm{~min}$, water-quenching, then artificial ageing at $160{ }^{\circ} \mathrm{C}$ for $10 \mathrm{~h}$ and air-cooling. The workpiece with a dimension of $550 \times 180 \times 4 \mathrm{~mm}$ was used in this study, which was machined from large $4 \mathrm{~mm}$ thick plates, and the width of the machined workpiece $(180 \mathrm{~mm})$ is parallel with the rolling direction.

Table 1. Main chemical composition of AA6082 (wt\%).

\begin{tabular}{|c|c|c|c|c|c|c|c|}
\hline $\mathrm{Al}$ & $\mathrm{Si}$ & $\mathrm{Mg}$ & $\mathrm{Mn}$ & $\mathrm{Fe}$ & $\mathrm{Cr}$ & $\mathrm{Zn}$ & $\mathrm{Cu}$ \\
\hline Balance & 0.82 & 0.82 & 0.64 & 0.24 & 0.09 & 0.024 & 0.021 \\
\hline
\end{tabular}

\subsection{Flexible tool}

The investigated flexible tool in this study was designed by Lam [10], as shown in Fig. 1(a) [11]. This flexible tool mainly consists of the top and bottom die holders, sparsely distributed forming pins, splines, and an elastometic sheet. More details about the tool design can be found in [11] and [12].

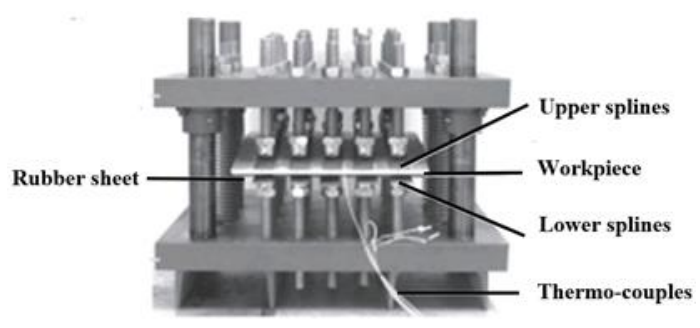

(a)

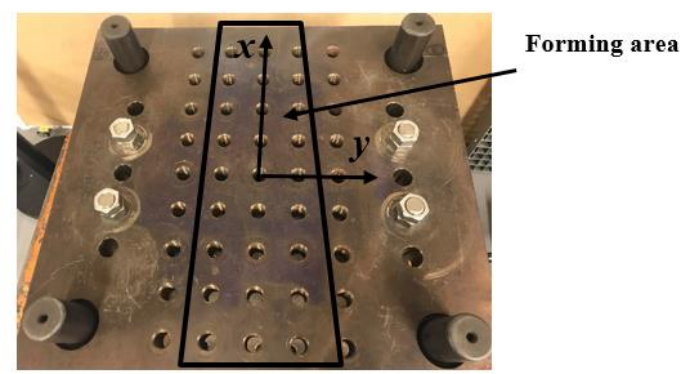

(b)
Fig. 1: (a) The flexible tool used for CAF and test setup [11]; (b) The top view of the tool without pins.

There are five rows of nine uniformly spaced holes on both the top and bottom die holders, where forming pins are located as shown in Fig. 1(b). The interval between each row and column of pins is $60 \mathrm{~mm}$. The number of pins can be changed according to the actual need, and the heights of pins can be adjusted, based on the target forming shape. In this study, three rows of pins in the centre of die holders were used as the forming area, as marked in Fig. 1(b).

A spline with a dimension of $500 \times 30 \times 3 \mathrm{~mm}$ is attached onto each row of pins in both upper and bottom die holders, to maintain the continuity of the surface generated by sparse pins. An elastomeric sheet (silicone rubber) with a dimension of $500 \times 300 \times 12 \mathrm{~mm}$ is placed between the workpiece and bottom splines to decrease the stress concentration on the plate, as shown in Fig. 1(a). Two materials of splines were used in this study, mild steel with a yield strength of $450 \mathrm{MPa}$ and $75 \mathrm{Cr} 1$ spring steel with a yield strength of $1000 \mathrm{MPa}$.

\subsection{Creep-age forming tests}

Four groups of CAF experiments were carried out to investigate the exact function of each part of the tool and the key factors in the multi-point flexible tool that affect the forming results, as shown in Table 2. The same tool shape, a single curved surface with a radius of $700 \mathrm{~mm}$ along the $x$ axis, was used for all CAF tests in this study.

Table 2: Details of four groups of CAF experiments.

\begin{tabular}{|c|c|c|}
\hline $\begin{array}{l}\text { Test } \\
\text { Group }\end{array}$ & Key factor & Test information \\
\hline 1 & $\begin{array}{c}\text { Interval } \\
\text { between pins }\end{array}$ & $\begin{array}{l}\text { (a) Five uniformly distributed } \\
\text { pins in each row }(5 \times 3) \text {, uniform } \\
\text { pin interval: } 120 \mathrm{~mm} \text {; } \\
\text { (b) Seven pins in each row }(7 \times 3) \text { : } \\
\text { pin interval: } 60 \text { and } 120 \mathrm{~mm} \text {; } \\
\text { (c) Nine uniformly distributed } \\
\text { pins in each row }(9 \times 3) \text {; pin } \\
\text { interval: } 60 \mathrm{~mm} \text {. (Fig. } 2)\end{array}$ \\
\hline 2 & $\begin{array}{c}\text { Arrangement } \\
\text { of pins }\end{array}$ & $\begin{array}{l}\text { (a) Asymmetric: nine pins at the } \\
\text { top, three pins at the bottom ( } 9 / 3 \text { ); } \\
\text { (b) Asymmetric: nine pins at the } \\
\text { top, five pins at the bottom ( } 9 / 5) \text {; } \\
\text { (c) Symmetric: nine pins at both } \\
\text { the top and bottom (9/9). (Fig. } 4)\end{array}$ \\
\hline 3 & $\begin{array}{c}\text { Accuracy of } \\
\text { the pin } \\
\text { height }\end{array}$ & $\begin{array}{l}\text { Same symmetric pins } \\
\text { arrangement }(7 \times 3) \text {. } \\
\pm 2 \mathrm{~mm} \text { error of height were } \\
\text { added to the pins in the } x+\text { area }(x \\
=+120,+180 \text { and }+240 \mathrm{~mm}) . \\
\text { (Fig. 6) }\end{array}$ \\
\hline
\end{tabular}




\begin{tabular}{|l|l|l|}
\hline 4 & $\begin{array}{l}\text { Material of } \\
\text { the splines }\end{array}$ & $\begin{array}{l}\text { Same symmetric pins } \\
\text { arrangement }(5 \times 3) \\
\text { (a) Mild steel; } \\
\text { (b) Spring steel. }\end{array}$ \\
\hline
\end{tabular}

The main procedures of the CAF tests include: (a) adjust the pin height to achieve the target tool surface, and prepare the whole set of flexible tool as shown in Fig. 1(a); (b) load the tool by a 250 ton Instron servo-hydraulic testing machine until the plate is deformed to the target shape (the same maximum load of $100 \mathrm{KN}$ was applied for all CAF tests); (c) use bolts and nuts to lock the tool, and release the pressure from Instron machine; (d) move the tool to a fan-assisted Aerotherm box oven and run the creep-ageing thermal cycle at $160{ }^{\circ} \mathrm{C}$ for 3 hours; (e) cool down, unload the tool, and let springback occur; (d) measure the dimensions of the deformed plate along its centre line $(x=0)$ with a Nikon LK G90C coordinate measuring machine (CMM).

\section{Results and discussion}

\subsection{Effects of the interval between pins}

Fig. 2 shows the pin number and interval details of the first group of test in Table 2. From five pins to nine pins in each row, the interval between pins decrease from 120 $\mathrm{mm}$ to $60 \mathrm{~mm}$.

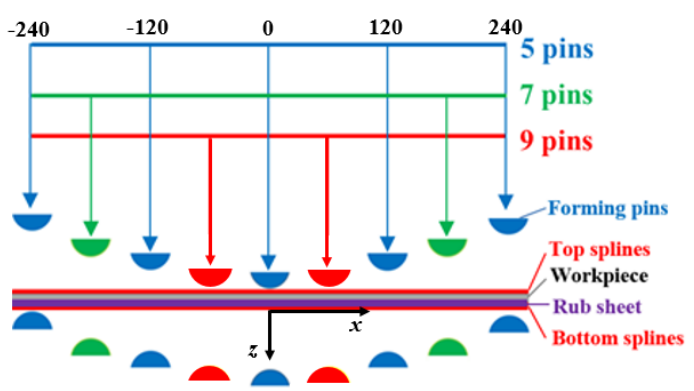

Fig. 2: Different pin number and interval conditions used in the first group of CAF tests.

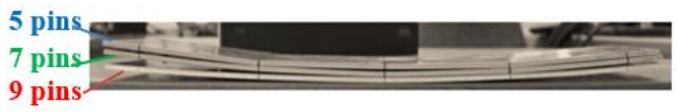

(a)

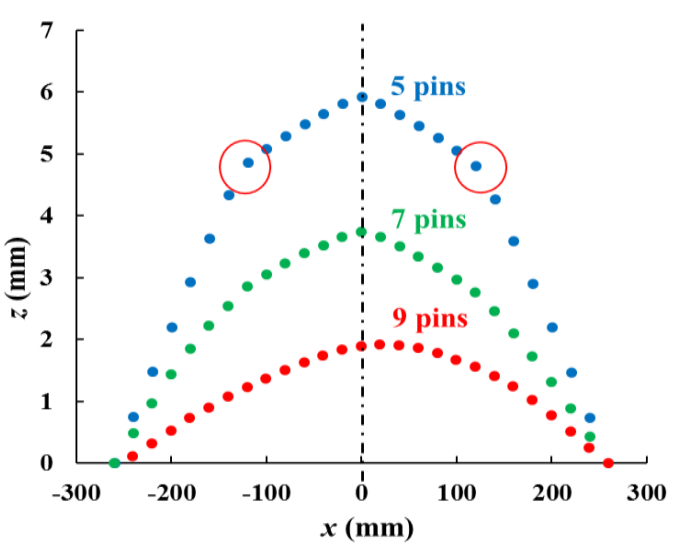

(b)

Fig. 3: (a) Side view of the CAFed plates under different pin interval conditions; (b) deflection results of the centre line $(x=$ $0)$ of CAFed plates obtained by CMM.

Fig. 3 shows the shape of the CAFed plates with different pins number and interval conditions. It can be observed from Fig. 3 that significant kinks (red circles in Fig. 3(b)) were present in the CAFed plate when using five forming pins in each row of the tool, gentle kinks occurred in the formed plate with seven forming pins in each row, while almost smooth curve was achieved in the formed plate when using nine pins. It is also found that with less forming pins, i.e., larger pin interval along the $x$ axis, larger deflections were achieved. From Fig. 3(b), it can be seen that kinks are mainly present around $x= \pm$ $120 \mathrm{~mm}$, where pins were located, indicating that the stress concentration may have occurred in the pin areas. As higher stresses were generated in these regions, more significant deformation (kinks) occurred. This can explain why smaller but less smooth curvature was generated by using more pins. It can be concluded that the interval between neighbouring pins can significantly affect the shape of the plates after CAF and smaller interval between pins generate a shape with smoother but smaller curvature; more pins can benefit the forming results, but make it more towards solid tools, reducing the benefit of flexible tool at the same time. The suitable interval between pins should be dependent on the target forming curvature, and further investigations are needed to quantify the exact relationship between the proper pin interval and the target forming curvature.

\subsection{Effects of the arrangement of pins}

Fig. 4 shows the three different arrangements of pins used for the CAF tests in this study: $9 / 3,9 / 5$, and $9 / 9$, as indicated in Table 2.

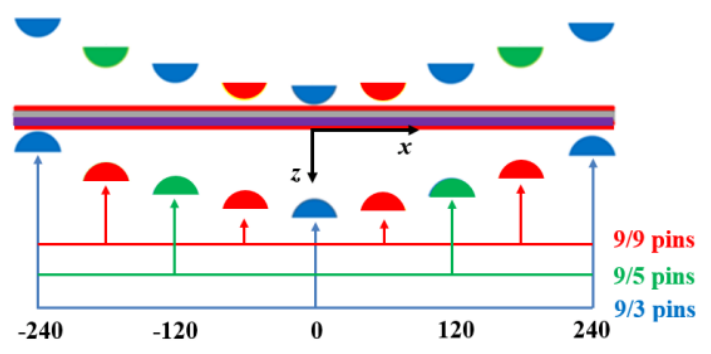


Fig. 4: Arrangement of pins: 9/3, 9/5 and 9/9.

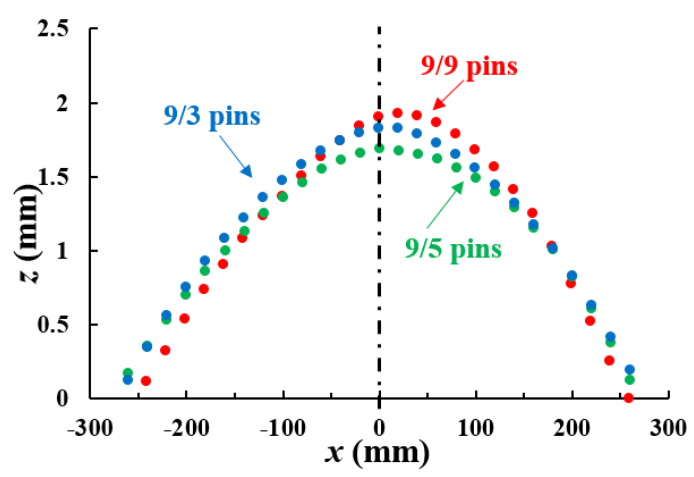

Fig. 5: Shape of the CAFed plates under three different arrangement of pins: $9 / 9,9 / 5$ and $9 / 3$.

Fig. 5 shows the surface profile along centre line $(x=0)$ of the plates after CAF under three different pins arrangements. It can be observed that there is slight difference among the final shape of the CAFed plates under these three different pins arrangements. It indicates that reducing the number of pins on one side will not largely affect the forming results. This proves the feasibility of using asymmetric number of pins in the multi-point flexible tool to deform the plates in the CAF process. Thus, for the future CAF tests, it is viable to decrease the number of pins on one side, which can reduce more weight and increase the efficiency.

\subsection{Effects of accuracy of pin height}

Errors of $\pm 2 \mathrm{~mm}$ errors were intentionally introduced to some target pin height in the $x+$ region, as shown in Fig. 6 , so as to investigate the sensitivity of the pin height accuracy on the formed shape.

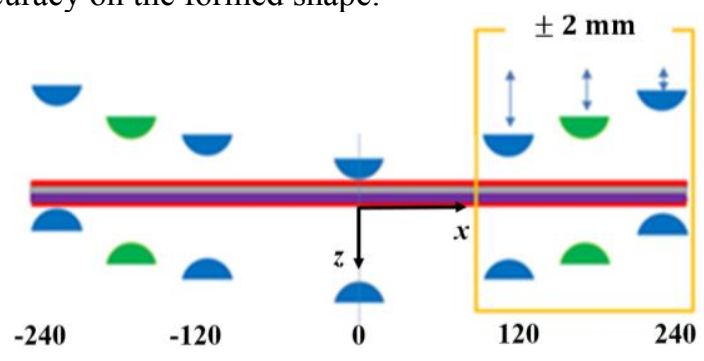

Fig. 6: Arrangement of pins: $\pm 2 \mathrm{~mm}$ errors added to the target pin height for the pins on the $x+$ direction.

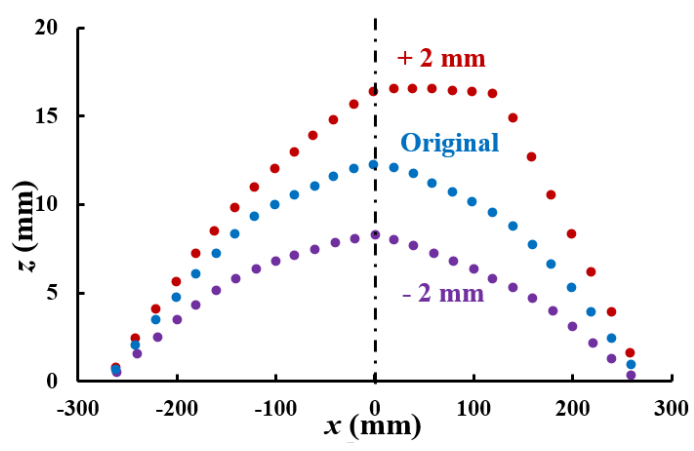

Fig. 7: Comparison of the shapes of CAFed plates between forming tools with original pin height and intentionally adjusted pin height with 2 and $-2 \mathrm{~mm}$ error added to the pins on $x+$ direction.

Fig. 7 shows the shape of the formed plates with different conditions, as configured in Fig. 6. A significant difference of final shape can be observed when $\pm 2 \mathrm{~mm}$ errors were introduced. It can also be seen that errors in $x+$ side affect the shape of the whole plate. Thus, it can be concluded that the final plastic deformation is quite sensitive to the pin height and small error in pin height can lead to significant difference of the final formed shape. As a result, during the set-up stage of the experiments, the pin height should be adjusted as accurate as possible to achieve best results.

\subsection{Effects of the material of splines}

Another component of the flexible tool for the CAF process is splines. The initial intention of adding splines between workpiece and pins is to keep the continuity of the surface of the tool and help to avoid indentations in the plate by reducing stress concentration. Thus, the material chosen for the spline needs to have sufficiently high strength to prevent it from deforming plastically. In this study, two materials were used for splines in the CAF tests: mild steel and $75 \mathrm{Cr} 1$ spring steel, which are much stronger than the $\mathrm{Al}$ alloy workpiece. The experimental results are shown in Fig. 8. Two cases were investigated: one is measured after loading, the other is after complete CAF process. It can be observed that larger deflections were produced when using mild steel splines. This is because the mild steel with lower strength is easier to deform rather than the other one. Smoother curves were obtained by using spring steel splines than mild steel splines. Additionally, visual examination of the splines of these two materials after $\mathrm{CAF}$, revealed that plastic deformation occurred on the mild steel splines, but no plastic deformation was present on the spring steel splines. The latter means that the spring steel splines can be repeatedly used which is beneficial for the running cost. As a result, to generate smoother shape, and take into consideration of reusability of the splines, spring steel is a better choice as the material of the splines.

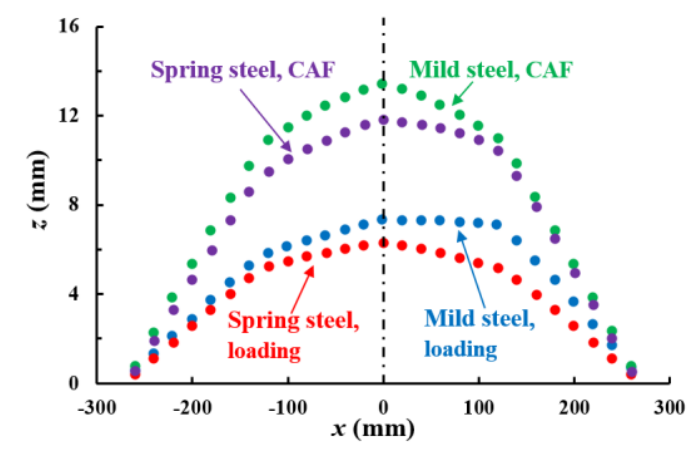

Fig. 8: Shape of the deformed plates after different process (CAF/ loading) with different material of the splines (mild steel/ spring steel).

\section{Conclusions}


In this study, four different groups of CAF tests related to the multi-point flexible tool were carried out to investigate the effects of the interval between pins, the asymmetric arrangement of pins, the error requirement of the pin height, and the material of splines on the forming results. The experimental study in this work helps to reveal how these key factors affect the forming results with flexible tool during the CAF process, providing a practical guidance on the setup of forming tools, and facilitating further applications of this kind of flexible tool in industry. The following conclusions can be drawn:

1. Reducing the interval between pins can efficiently smooth the shape of the plates after CAF, but decrease the achieved deflections. When the target curvature is large and complex, small interval between pins is preferred to avoid kinks.

2. The feasibility of asymmetric arrangement of pins for CAF has been proven, which means one-to-one correspondence between pins at top and bottom is not necessary. Asymmetric arrangement of pins can largely decrease the number of used pins, reduce the weight of tool, and increase the efficiency.

3. The forming results are very sensitive to the pin height, and the error of pin height should be carefully controlled. Otherwise a large difference between the final forming shape and the target shape will occur.

4. Compared with mild steel, spring steel, which has higher strength, is more suitable as the spline material. It can help to generate smoother shape of the deformed plate and can be used after CAF process.

\section{Acknowledgements}

Much appreciated is the strong support received from CRRC Qingdao Sifang Co., Ltd. The research was performed at the CRRC Sifang-Imperial Centre for Rail Transportation Manufacturing Technologies at Imperial College London.

\section{References}

1. L. Zhan, J. Lin, T. Dean. Int. J. of Mech. Tool. Manu. 51, 1 (2011) 1-17.

2. K. Watcham. Mate. World. 12 (2004) 10-11.

3. M. Holman. J. of Manu. Working Tech. 20 (1989) 477-488.

4. A. Levers, J. of Manu. Eng. 82 (2003) 42-45.

5. A. Levers, PAT US 7,322,223 B2, 29 Jan 2008.

6. A. Levers, Int. J. for History of Eng. \& Tech. 80 (2010) 55-79.

7. H. Bornschlegl, PAT US 6,264,771. 24 Jul 2001.

8. TL. Newkirk, MC Holman, PAT US5, 729,462, 17 Mar 1998.

9. HM. Brewer, PAT US 5, 168, 169, 1 Dec 1992.

10. A. Lam, Z. Shi, J. Lin, X. Huang, Y. Zeng, T. Dean. Int. J. of Adv. Manu. Tech. 80 (2015) 361-372.
11. A. Lam, Z. Shi, J. Lin, X. Huang. Int. J. of Mech. Sci. 103 (2015) 115-126.

12. A. Lam, PhD thesis: A flexible tool design and integrated modelling techniques for springback compensation in creep age forming. (2016) 